The Quarterly Journal of Mathematics Advance Access published on May 29, 2007

Quart. J. Math. 58 (2007), 297-311; doi:10.1093/qmath/ham013

\title{
SPECIAL SYMPLECTIC SIX-MANIFOLDS
} by DIEGO CONTI ${ }^{\dagger}$

(Dipartimento di Matematica e Applicazioni, Università di Milano Bicocca, Via Cozzi 53, 20125 Milano, Italy)

and ADRIANO TOMASSINI

(Dipartimento di Matematica, Università di Parma, Via G.P. Usberti 53/A, 43100 Parma, Italy)

[Received 16 December 2006. Revised 30 January 2007]

\begin{abstract}
We classify nilmanifolds with an invariant symplectic half-flat structure. We study the transverse or quotient geometry of six-manifolds with an SU(3)-structure preserved by a Killing vector field, giving characterizations in the symplectic half-flat and integrable case.
\end{abstract}

\section{Introduction}

A half-flat manifold is a six-dimensional manifold endowed with an SU(3)-structure with intrinsic torsion that is symmetric. An SU(3)-structure defines a non-degenerate two-form $\omega$, an almost-complex structure $J$ and a complex volume form $\Psi$; the half-flat condition is equivalent to requiring $\omega \wedge \omega$ and the real part of $\Psi$ be closed [4]. Hypersurfaces in seven-dimensional manifolds with holonomy $G_{2}$ have a natural half-flat structure, given by the restriction of the holonomy group representation; the intrinsic torsion can then be identified with the second fundamental form. Conversely, every real analytic half-flat manifold $(M, \omega, \Psi)$, can be embedded isometrically as a hypersurface in a manifold with holonomy contained in $G_{2}$. Writing the $G_{2}$ metric explicitly is a matter of solving certain evolution equations. Hitchin [11] proved that such a solution can be viewed as an integral line for a Hamiltonian flow on an infinite-dimensional symplectic space, namely the product of cohomology classes $\left[\omega^{2}\right] \times[\mathfrak{R e} \Psi]$.

Given a six-manifold $M$ with an SU(3)-structure, one can consider the product $G_{2}$-structure on $M \times S^{1}$. Half-flat manifolds satisfying special conditions on this product $G_{2}$-structure have been studied: in [3, 5], the six-dimensional nilmanifolds carrying invariant SU(3)-structures of these types have been classified. More generally, the problem of classifying nilmanifolds admitting invariant half-flat structures is open.

In this paper, we focus on the symplectic case; that is to say, we take into consideration half-flat structures for which $\omega$ is closed. Symplectic half-flat manifolds can also be viewed as symplectic manifolds $(M, \omega)$ endowed with two extra objects, namely an $\omega$-calibrated almost-complex structure and a $(3,0)$-form with closed real part which is parallel with respect to the Chern connection $[7,8]$.

\footnotetext{
†Corresponding author. E-mail: diego.conti@unimib.it

†E-mail: adriano.tomassini@unipr.it
} 
The evolution flow mentioned above is transverse to the space of symplectic half-flat structures, except at its critical points, that correspond to integrable (namely, Calabi-Yau) SU(3)-structures.

More generally, we consider arbitrary SU(3)-structures on six-manifolds, and we investigate the geometry determined by a Killing vector field that preserves such a structure. A similar situation has been studied in [1], in the context of seven-dimensional manifolds with holonomy $\mathrm{G}_{2}$. A special case has been studied in [12], where it was proved that there is only one complete example of a nearlyKähler SU(3)-structure with a Killing vector field of unit norm, namely the standard nearly-Kähler structure on $S^{3} \times S^{3}$.

The paper is divided into two parts. In the first part, we classify nilmanifolds carrying an invariant symplectic half-flat structure; the special case $b_{1} \geq 4$ was carried out by Bedulli in [2]. Since six-dimensional nilmanifolds can be realized as circle bundles over nilmanifolds of dimension five, this classification problem can be reduced to a problem in five dimensions: indeed, the symplectic half-flat structure induces an SU(2)-structure on the base of the circle bundle, satisfying certain conditions involving the curvature (see Lemma 2.2). In Lemma 2.3, we classify the five-dimensional nilmanifolds with this type of induced structure. We then use this lemma to show that every six-dimensional nilmanifold admitting a symplectic half-flat structure is modelled on one of a list of three Lie algebras (Theorem 2.4). The corresponding nilmanifolds are the torus, a torus bundle over the four-dimensional torus (see [8, 9]) and a torus bundle over a three-dimensional torus (see $[2,9]$ ). In contrast with the first two, the third example is irreducible; moreover, the fibres of the torus fibration are special Lagrangian submanifolds.

In the second part, we generalize this situation and the construction of Lemma 2.2 to the non-invariant case. More precisely, we consider a six-manifold with an SU(3)-structure and a nowhere-vanishing Killing vector field preserving the structure. This vector field generates a rank one foliation, that we can assume regular, working locally. Then the leaf space is a five-dimensional manifold. If the leaves are compact there is a circle action on the six-manifold, and the leaf space is a quotient on which the curvature two-form is defined; in fact, a 'curvature' two-form can always be defined on the leaf space using the O'Neill tensor. The geometric structure we are interested in is an SU(2)-structure induced on the leaf space or quotient. The metric underlying this $\mathrm{SU}(2)$-structure is not the same as the quotient metric, but it is obtained from the quotient metric by rescaling along certain directions, as in [1]. We compute the intrinsic torsion of the SU(2)structure in terms of the intrinsic torsion of the SU(3)-structure, norm of the Killing vector field and curvature two-form (Proposition 3.1). As far as we know, this is the first detailed application of intrinsic torsion for SU(2)-structures on five-manifolds. We then characterize the symplectic halfflat manifolds in terms of the quotient structure (Proposition 3.2). In the case that the invariant SU(3)-structure is integrable, we obtain a stronger result: indeed, the intrinsic torsion of the quotient structure is determined by the norm of the Killing vector field, and so is the curvature form (Theorem 3.3).

\section{Invariant structures on nilmanifolds}

In this section, we introduce half-flat structures on six-manifolds and classify invariant symplectic half-flat structures on six-dimensional nilmanifolds.

Let $M$ be a six-dimensional manifold. An SU(3)-structure on $M$ is a pair $(\omega, \Psi)$, where $\omega$ is a nondegenerate two-form and $\Psi$ is a locally decomposable complex three-form, such that the following 
compatibility conditions hold:

$$
\begin{aligned}
\Psi \wedge \omega & =0, \\
\Psi \wedge \bar{\Psi} & =\frac{4}{3} i \omega^{3},
\end{aligned}
$$

and the almost-complex structure induced by $J$ is $\omega$-tamed. Recall that a decomposable complex three-form $\Psi=\theta^{1} \wedge \theta^{2} \wedge \theta^{3}$ determines an almost-complex structure by the condition that $\theta^{1}, \theta^{2}$ and $\theta^{3}$ span the space of forms of type $(1,0)$. The first condition asserts that $\omega$ is of type $(1,1)$, so $J$ is actually calibrated by $\omega$.

At each point $x$ of $M$, the three-form $\Psi_{x}$ has stabilizer conjugate to $\operatorname{SL}(3, \mathbb{C})$ for the natural action of $\mathrm{GL}^{+}\left(T_{x} M\right)$; on the other hand, the stabilizer of $\omega_{x}$ is conjugate to the symplectic group $\operatorname{Sp}(3, \mathbb{R})$ for the natural action of $\operatorname{GL}\left(T_{x} M\right)$. The compatibility conditions ensure that the intersection of the two stabilizers is conjugate to $\mathrm{SU}(3)$, so that an SU(3)-structure is defined.

We shall denote by $\psi^{+}$and $\psi^{-}$the real and imaginary parts of $\Psi$, respectively. It was shown by Hitchin [10] that having fixed the orientation, the real three-form $\psi^{+}$is sufficient to determine the almost-complex structure $J$ and therefore $\psi^{-}$. Thus, an $\operatorname{SU}(3)$-structure $(\omega, \Psi)$ is really determined by the pair $\left(\omega, \psi^{+}\right)$.

We now introduce a special class of SU(3)-structures on six-manifolds, related to sevendimensional Riemannian manifolds with holonomy contained in $G_{2}$ [4].

Definition 2.1: An SU(3)-structure $\left(\omega, \psi^{+}\right)$on a six-manifold is half-flat if $\omega \wedge \omega$ and $\psi^{+}$are closed.

The two-form $\omega$ appearing in the characterization of SU(3)-structures is required to be nondegenerate; if it is also closed, it defines a symplectic structure. In this case, we say that the SU(3)structure is symplectic.

Consider a nilmanifold $M$, that is, a compact manifold of the form $\Gamma \backslash G$, where $G$ is a sixdimensional nilpotent group with Lie algebra $\mathfrak{g}$ and $\Gamma$ a uniform discrete subgroup of $G$. Recall that in six dimensions, every nilpotent Lie algebra $\mathfrak{g}$ gives rise to such a nilmanifold.

We say that a structure on the nilmanifold $M$ is invariant if it pulls back to a left-invariant structure on $G$. Invariant structures can be viewed as structures on the Lie algebra $\mathfrak{g}$; using the above characterization of SU(3)-structures in terms of differential forms, we shall mainly work with the dual $\mathfrak{g}^{*}$.

We start by reducing the problem to a problem in five dimensions; the idea is to realize $M$ as a circle bundle over a five-dimensional manifold, in such a way that the SU(3)-structure on $M$ is invariant under the circle action. The geometry of this construction will be studied in Section 3; here, we shall use the following algebraic result.

LEMMA 2.2 Let $\left(\omega, \psi^{+}\right)$be a symplectic half-flat structure on a nilpotent Lie algebra $\mathfrak{g} ;$ we have an orthogonal decomposition

$$
\mathfrak{g}^{*}=\langle\eta\rangle \oplus V^{5}
$$

where $\eta$ is a unit form, and

$$
d\left(\mathfrak{g}^{*}\right) \subseteq \Lambda^{2} V^{5} .
$$


Define forms $\alpha, \omega_{1}, \omega_{2}$ and $\omega_{3}$ on $\operatorname{ker} \eta$ by

$$
\begin{aligned}
& \omega=\omega_{3}+\eta \wedge \alpha, \\
& \Psi=\left(\omega_{1}+i \omega_{2}\right) \wedge(\eta+i \alpha) .
\end{aligned}
$$

Setting $\phi=d \eta$, the following hold:

$$
\begin{aligned}
d \alpha & =0, \quad d \omega_{1}=0, \\
d \omega_{3} & =-\phi \wedge \alpha, \quad d\left(\omega_{2} \wedge \alpha\right)=\omega_{1} \wedge \phi .
\end{aligned}
$$

Proof. By Engel's theorem, some non-zero $\xi$ in $\mathfrak{g}$ satisfies

$$
\operatorname{ad}(\xi)=0 .
$$

Choosing for $\eta$ a suitable multiple of $\xi^{b}$ and setting $V^{5}=\eta^{\perp}$, the first part of the lemma is satisfied. By definition

$$
0=d \omega=d \omega_{3}+\phi \wedge \alpha-\eta \wedge d \alpha
$$

isolating the component in $\eta \wedge \Lambda^{2} V^{5}$, we deduce that $\alpha$ is closed and $d \omega_{3}$ satisfies the required equation.

Similarly, the rest of (3) follows from

$$
0=d \psi^{+}=d \omega_{1} \wedge \eta+\omega_{1} \wedge \phi-d\left(\omega_{2} \wedge \alpha\right)
$$

REMARK: The forms $\left(\alpha, \omega_{i}\right)$ introduced in Lemma 2.2 define an SU(2)-structure on $\mathfrak{g}$. More generally, we recall that differential forms $\left(\alpha, \omega_{1}, \omega_{2}, \omega_{3}\right)$ on a five-manifold define an SU(2)-structure if and only if at each point (and hence locally), there exists a coframe $e^{1}, \ldots, e^{5}$ such that

$$
\begin{gathered}
\alpha=e^{5}, \quad \omega_{1}=e^{12}+e^{34}, \\
\omega_{2}=e^{13}+e^{42}, \quad \omega_{3}=e^{14}+e^{23} .
\end{gathered}
$$

Here and in the sequel, $e^{12}$ is short for $e^{1} \wedge e^{2}$ and so on. If one fixes an orientation, the condition above is equivalent to the existence of a triplet $\left(\omega_{1}, \psi_{2}, \psi_{3}\right)$ with

$$
\omega_{1}=e^{12}+e^{34}, \quad \psi_{2}=e^{135}+e^{425}, \quad \psi_{3}=e^{145}+e^{235} .
$$

By construction, $V^{5}$ is itself the dual of a nilpotent Lie algebra; we shall proceed by listing the five-dimensional Lie algebras that arise this way. To describe Lie algebras, we shall use symbolic expressions such as

$$
\mathfrak{g}=(0,0,0,0,0,12)
$$

meaning that $\mathfrak{g}^{*}$ has a basis $\eta^{1}, \ldots, \eta^{6}$ such that $d \eta^{6}=\eta^{1} \wedge \eta^{2}$, and $\eta^{i}$ is closed for $i=1, \ldots, 5$.

LEMMA 2.3 In the hypotheses of Lemma $2.2, V^{5}$ is one of

$$
(0,0,0,0,0),(0,0,0,0,12),(0,0,0,12,13) .
$$


Proof. By the same argument we used in the proof of Lemma 2.2, we can construct a filtration

$$
V^{0} \subset \cdots \subset V^{5}, \quad \text { where } \operatorname{dim} V^{i}=i, d V^{i+1} \subset \Lambda^{2} V^{i} .
$$

We can assume that ker $d$ coincides with some $V^{i}$. Moreover, by Lemma 2.2, we can assume that $\alpha$ lies in $V^{1} \subset V^{4}$; therefore, using the fact that $\mathrm{SU}(2)$ acts transitively on the three-dimensional sphere, we can fix a basis $e^{1}, \ldots, e^{5}$ of $V^{5}$ satisfying (4), with $e^{4}$ in $\left(V^{4}\right)^{\perp}$.

We will show that the operator $d: V^{5} \rightarrow \Lambda^{2} V^{5}$ satisfies

$$
\operatorname{ker} d \supset V^{3}, \quad d\left(V^{5}\right) \subset \Lambda^{2} V^{3} .
$$

Then it will follow from the classification of five-dimensional nilpotent Lie algebras that $V^{5}$ is one of the Lie algebras appearing in the statement.

By Lemma 2.2

$$
0=d e^{12}+d e^{34} \equiv d e^{3} \wedge e^{4} \bmod \Lambda^{2} V^{4},
$$

implying that $e^{3}$ is closed. Thus, we can assume

$$
V^{2}=\left\langle e^{3}, e^{5}\right\rangle ; \quad V^{4}=\left\langle e^{1}, e^{2}, e^{3}, e^{5}\right\rangle
$$

Define a real constant $h$, a two-form $\gamma \in \Lambda^{2}\left\langle e^{1}, e^{2}, e^{3}\right\rangle$ and one-forms $\phi_{4}, \phi_{5}$ in $\left\langle e^{1}, e^{2}, e^{3}\right\rangle$, such that

$$
\phi=\phi_{5} \wedge e^{5}+\phi_{4} \wedge e^{4}+h e^{45}+\gamma
$$

Since $\phi$ is closed,

$$
d \phi_{5} \wedge e^{5}-\phi_{4} \wedge d e^{4}+h d e^{4} \wedge e^{5}+d \gamma=-d \phi_{4} \wedge e^{4}
$$

the left-hand side lies in $\Lambda^{3} V^{4}$, that is, it has no component containing $e^{4}$, so both sides are zero and $d \phi_{4}=0$.

By Lemma $2.2 d \omega_{3}=-\phi \wedge \alpha$, giving

$$
d e^{14}+d e^{2} \wedge e^{3}=-\phi_{4} \wedge e^{45}-\gamma \wedge e^{5}
$$

comparing the components in $e^{4} \wedge \Lambda^{2} V^{4}$, we obtain

$$
d e^{1}=\phi_{4} \wedge e^{5}
$$

Again by Lemma 2.2, $d \psi_{2}=\omega_{1} \wedge \phi$; on the other hand, $d \psi_{2}=d \omega_{2} \wedge \alpha$, so we can drop the component of $\phi$ not containing $e^{5}$ and write

$$
\left(d e^{13}+d e^{42}\right) \wedge e^{5}=\left(e^{12}+e^{34}\right) \wedge\left(\phi_{5} \wedge e^{5}+h e^{45}\right) .
$$

The components containing $e^{4}$ give

$$
d e^{2} \wedge e^{5}=e^{3} \wedge \phi_{5} \wedge e^{5}-h e^{125}
$$


and wedging with $e^{3}$,

$$
d e^{2} \wedge e^{35}=-h e^{1235} .
$$

Since $d e^{2}$ is in $\Lambda^{2} V^{3}$, the left-hand side is in $\Lambda^{4} V^{3}$, and so it is zero. We conclude that $h=0$ and

$$
d e^{2} \wedge e^{5}=e^{3} \wedge \phi_{5} \wedge e^{5}
$$

Now, either $V^{3}=\left\langle e^{1}, e^{3}, e^{5}\right\rangle$, or some linear combination $\lambda e^{1}+e^{2}$ lies in $V^{3}$ and consequently $0=\left(\lambda d e^{1}+d e^{2}\right) \wedge e^{5}=d e^{2} \wedge e^{5}$. Either way,

$$
\phi_{5} \in\left\langle e^{1}, e^{3}\right\rangle .
$$

By Lemma $2.2 \omega_{1}$ is closed, giving

$$
\phi_{4} \wedge e^{52}-e^{1} \wedge d e^{2}=e^{3} \wedge d e^{4} .
$$

In order to prove (5), we must distinguish three cases.

(i) Suppose that $\phi_{4}$ is not a multiple of $e^{3}$; then

$$
V^{3}=\left\langle e^{3}, e^{5}, \phi_{4}\right\rangle
$$

and $d$ is zero on $V^{3}$. Moreover $e^{1}$ is not closed, so $V^{4}=V^{3} \oplus\left\langle e^{1}\right\rangle$. Since $e^{2}$ is in $V^{4}$, we have $d e^{2}=k e^{5} \wedge \phi_{4}$ for some (possibly zero) constant $k$. So (10) becomes

$$
\phi_{4} \wedge e^{52}-k e^{15} \wedge \phi_{4}=e^{3} \wedge d e^{4}
$$

implying that

$$
d e^{4} \wedge e^{3} \wedge \phi_{4}=0=d e^{4} \wedge e^{35}
$$

Since the space of closed two-forms in $\Lambda^{2} V^{4}$ is

$$
\Lambda^{2} V^{3} \oplus\left\langle e^{15}, e^{1} \wedge \phi_{4}\right\rangle
$$

we can conclude that $d e^{4}$ lies in $\Lambda^{2} V^{3}$; we already know that $d\left(V^{3}\right)=0$, so there is nothing left to prove in this case.

In general, the component of (7) in $\Lambda^{3} V^{4}$ gives

$$
-e^{1} \wedge d e^{4}+d e^{2} \wedge e^{3}=-\gamma \wedge e^{5}
$$

in particular, $d e^{4} \wedge e^{15}=0$. Moreover, we can rewrite (6) as

$$
-\phi_{4} \wedge d e^{4}+d \gamma=0
$$

(ii) Suppose now that $\phi_{4}=0$; then $e^{1}$ is closed and we can assume that $V^{3}=\left\langle e^{1}, e^{3}, e^{5}\right\rangle$. By (10)

$$
-e^{1} \wedge d e^{2}=e^{3} \wedge d e^{4}
$$

so clearly $d e^{4} \wedge e^{13}=0$; moreover $d e^{4} \wedge e^{35}=0$, since by (9) $d e^{2} \wedge e^{15}$ is zero. It follows that $d e^{4}$ lies in $\Lambda^{2} V^{3}$, completing the proof in this case. 
(iii) The remaining case is the one where $\phi_{4}=a e^{3}$ for some non-zero $a$. By (12) and (10), this condition implies

$$
d \gamma=a e^{3} \wedge d e^{4}=a^{2} e^{352}-a e^{1} \wedge d e^{2} .
$$

Equations (9) and (10) show that

$$
d e^{2} \wedge e^{15}=0=d e^{2} \wedge e^{13}=d e^{2} \wedge e^{35}
$$

so $d e^{2}$ lies in $\Lambda^{2}\left\langle e^{1}, e^{3}, e^{5}\right\rangle$. Hence the space of closed forms in $\Lambda^{2} V^{4}$ is contained in

$$
\Lambda^{2}\left\langle e^{1}, e^{3}, e^{5}\right\rangle \oplus e^{2} \wedge\left\langle e^{3}, e^{5}\right\rangle
$$

wedging the closed two-form $\gamma-a e^{12}$ with $e^{35}$ we deduce

$$
\gamma \wedge e^{35}=a e^{1235}
$$

Comparing with (11), we find $e^{13} \wedge d e^{4}=-a e^{1235}$, which together with (13) gives the contradiction

$$
-a^{2} e^{1235}=a^{2} e^{1352}
$$

All three possibilities listed in Lemma 2.3 can occur.

- On $V^{5}=(0,0,0,0,0)$, set

$$
\omega_{1}=\eta^{12}+\eta^{34}, \quad \psi_{2}=\eta^{135}+\eta^{425}, \quad \psi_{3}=\eta^{145}+\eta^{235}, \quad \phi=0 .
$$

- On $V^{5}=(0,0,0,0,12)$, set

$$
\omega_{1}=\eta^{34}+\eta^{15}, \quad \psi_{2}=\eta^{312}+\eta^{542}, \quad \psi_{3}=\eta^{352}+\eta^{412}, \quad \phi=-\eta^{13} .
$$

- On $V^{5}=(0,0,0,12,13)$, set

$$
\begin{array}{ll}
\omega_{1}=\eta^{24}+\eta^{35}, & \psi_{2}=\eta^{123}+\eta^{154}, \quad \psi_{3}=\eta^{125}+\eta^{143}, \quad \phi=-2 \eta^{23} ; \quad \text { or } \\
\omega_{1}=\eta^{24}-\eta^{35}, & \psi_{2}=-\eta^{123}+\eta^{154}, \quad \psi_{3}=\eta^{125}-\eta^{143}, \quad \phi=0 .
\end{array}
$$

It is easy to verify that equations (3) are satisfied in these cases. The construction can then be inverted: define

$$
\mathfrak{g}^{*}=\langle\eta\rangle \oplus V^{5}
$$

declaring that $d \eta=\phi$; clearly, $\mathfrak{g}^{*}$ is the dual of a nilpotent Lie algebra $\mathfrak{g}$. A straightforward calculation shows that the SU(3)-structure on $\mathfrak{g}$ defined by (2) is half-flat and symplectic.

So, there are at least three non-isomorphic nilpotent Lie algebras that admit a symplectic half-flat structure. It only remains to show that this list is complete. Indeed, we prove the following.

THEOREM 2.4 The six-dimensional nilpotent Lie algebras whose corresponding nilmanifold carries an invariant symplectic half-flat structure are

$$
(0,0,0,0,0,0), \quad(0,0,0,0,12,13), \quad(0,0,0,12,13,23) .
$$


Proof. We retain the notation from the proof of Lemma 2.3. We first show that $\phi_{4}$ is zero; in other words, case (i) of Lemma 2.3 cannot occur, like case (iii), which we have already ruled out. Indeed, suppose that $\phi_{4}$ is independent of $e^{3}$ and $e^{5}$. Hence $V^{5}=(0,0,0,12,13)$; indeed, $d e^{1}$ and $d e^{4}$ must be independent, since otherwise a combination of $e^{1}$ and $e^{4}$ would lie in ker $d$, which is orthogonal to $e^{4}$ by construction.

Observe that $\left\langle e^{1}, e^{3}, \phi^{4}\right\rangle$ has dimension three, because $\phi_{4}$ is closed but $e^{1}$ is not, and we are assuming that $\phi_{4}$ is not a multiple of $e^{3}$. Therefore

$$
\left\langle e^{1}, e^{3}, \phi^{4}\right\rangle=\left\langle e^{1}, e^{2}, e^{3}\right\rangle
$$

and we can write $\gamma=a e^{13}+b e^{1} \wedge \phi_{4}+c e^{3} \wedge \phi_{4}$. Equation (11) then yields

$$
-e^{1} \wedge d e^{4}+k e^{5} \wedge \phi_{4} \wedge e^{3}=-a e^{135}+b e^{15} \wedge \phi_{4}+c e^{35} \wedge \phi_{4},
$$

so in particular

$$
d e^{4}=a e^{35}-b e^{5} \wedge \phi_{4}
$$

Substituting in (12), it follows that

$$
-a \phi_{4} \wedge e^{35}+a \phi_{4} \wedge e^{53}=0
$$

that is, $a=0$; but then $d e^{4}=b e^{5} \wedge \phi_{4}$ is a multiple of $d e^{1}$, which is absurd.

We have proved that $\phi_{4}$ is necessarily zero; now assume that $e^{2}$ is closed. Then (10) and (11) give

$$
e^{3} \wedge d e^{4}=0, \quad e^{1} \wedge d e^{4}=\gamma \wedge e^{5}
$$

It follows that $d e^{4}=\lambda e^{35}+\mu e^{13}$ and $\gamma=\lambda e^{13}$ for some constants $\lambda$ and $\mu$. The components of (8) not containing $e^{4}$ give

$$
\mu e^{1325}=-e^{125} \wedge \phi_{5}
$$

Since as a consequence of (9) $\phi_{5}$ is a multiple of $e^{3}$, it follows that $\phi_{5}=-\mu e^{3}$. Summing up,

$$
\phi=-\mu e^{35}+\lambda e^{13}
$$

so that $\phi$ and $d e^{4}$ are either linearly independent or both zero. The resulting six-dimensional Lie algebras are

$$
(0,0,0,0,12,13), \quad(0,0,0,0,0,0) .
$$

If $e^{2}$ is not closed, $V^{5}$ is $(0,0,0,12,13)$. As both $d e^{4}$ and $d e^{2}$ are in $\Lambda^{2} V^{3},(11)$ implies that $\gamma$ is a multiple of $e^{13}$. Therefore, $\phi$ lies in $\Lambda^{2} V^{3}$ as well, forcing $\mathfrak{g}$ to be either $(0,0,0,12,13,23)$ or $(0,0,0,0,12,13)$.

For the convenience of the reader, we write down explicitly examples of symplectic halfflat structures on the nilmanifolds associated to the Lie algebras of Theorem 2.4. Consistently with Lemma 2.2, we apply (2) to the examples of page 4 . With notation as introduced 
before Lemma 2.3:

(1) On $\mathfrak{g}=(0,0,0,0,12,13)$, we set

$$
\omega=\eta^{35}+\eta^{41}-\eta^{62}, \quad \Psi=\left(\eta^{3}+i \eta^{5}\right) \wedge\left(\eta^{4}+i \eta^{1}\right) \wedge\left(-\eta^{6}+i \eta^{2}\right)
$$

then $d \omega=0=d \psi^{+}$. Similarly,

$$
\omega=\eta^{25}-\eta^{43}+\eta^{61}, \quad \Psi=\left(\eta^{2}+i \eta^{5}\right) \wedge\left(\eta^{4}-i \eta^{3}\right) \wedge\left(\eta^{6}+i \eta^{1}\right)
$$

is a symplectic half-flat structure on $\mathfrak{g}$.

(2) On $\mathfrak{g}=(0,0,0,12,13,23)$, we set

$$
\omega=\eta^{25}+\eta^{43}-\frac{1}{2} \eta^{61}, \quad \Psi=\left(\eta^{2}+i \eta^{5}\right) \wedge\left(\eta^{4}+i \eta^{3}\right) \wedge\left(-\frac{1}{2} \eta^{6}+i \eta^{1}\right) .
$$

Again, $d \omega=0=d \psi^{+}$.

\section{Circle bundles, quotients and intrinsic torsion}

In this section, we pursue an idea introduced in Section 2, namely that of reducing a six-dimensional manifold to a five-dimensional manifold by means of a quotient, and establishing a relation between the two geometries in terms of $G$-structures. Here we work in a more general context, without requiring invariance under a transitive action; however, for the construction to make sense we still need invariance along one direction, that is, a Killing vector field $X$ on the six-manifold. Then the directions orthogonal to $X$ give a rank 5 distribution on which an SU(2)-structure is induced. Thus, if $X$ is regular, the leaf space is a five-dimensional manifold with what we call the quotient $\mathrm{SU}(2)$-structure. Otherwise, this description is valid only locally.

We are interested in the intrinsic torsion of this induced SU(2)-structure. In particular, we characterize the intrinsic torsion of the SU(2)-structures obtained by taking the quotient of a symplectic half-flat structure, generalizing Lemma 2.2. Then, in the assumption that the starting SU(3)-structure is integrable, we write down a differential equation that the norm $t$ of the Killing vector field must satisfy and prove that the intrinsic torsion of the quotient structure depends only on $t$. More precisely, we give necessary and sufficient conditions on $\left(N, \alpha, \omega_{i}, t\right)$ for it to arise, locally, as the quotient of a six-manifold with an integrable SU(3)-structure. Observe that in the integrable case the six-manifold cannot be compact, unless it is reducible.

We consider objects of the form $\left(M, \omega, \psi^{+}, X\right)$, where $M$ is a six-dimensional manifold, $\left(\omega, \psi^{+}\right)$ is an SU(3)-structure on $M$ and $X$ is a regular vector field on $M$ which preserves the $\mathrm{SU}(3)$-structure, that is,

$$
\mathcal{L}_{X} \omega=0=\mathcal{L}_{X} \psi^{+} .
$$

The space of integral lines of $X$ is a five-manifold $N$. Since $X$ is a Killing vector field, the norm of $X$ is constant along the integral lines, and so defines a smooth function $t$ on $N$. Let $\eta$ be the the dual form to $X$, rescaled so that $\eta(X)=1$; set $\phi=d \eta$. It is easy to check that

$$
\left.\mathcal{L}_{X} \phi=0, \quad X\right\lrcorner \phi=0 .
$$

In general, a form $\phi$ on $M$ satisfying (14) is said to be basic, and it is the pullback of a form on $N$. In the sequel, we shall identify basic forms on $M$ with forms on $N$. Note that if $M$ is a circle bundle 
over $N$, its Chern class is represented by $1 / 2 \pi$. Now define forms $\left(\alpha, \omega_{i}\right)$ on $M$ by

$$
\begin{aligned}
& \left.\alpha=X\lrcorner \omega, \quad \omega_{3}=t X\right\lrcorner(\omega \wedge \eta), \\
& \left.\left.\omega_{1}=X\right\lrcorner \psi^{+}, \quad \omega_{2}=X\right\lrcorner \psi^{-} .
\end{aligned}
$$

By construction, all the forms appearing on the right-hand side of (15) are basic.

Now choose a local orthonormal basis of 1 -forms

$$
\frac{1}{\sqrt{t}} e^{1}, \ldots, \frac{1}{\sqrt{t}} e^{4}, \frac{1}{t} e^{5}, e^{6}
$$

on $M$, with $\eta=t^{-1} e^{6}$, such that

$$
\omega=\frac{1}{t}\left(e^{14}+e^{23}+e^{65}\right), \quad \Psi=\frac{1}{t}\left(e^{1}+i e^{4}\right) \wedge\left(e^{2}+i e^{3}\right) \wedge\left(e^{6}+\frac{1}{t} i e^{5}\right) .
$$

Then (4) is satisfied, so $\left(\alpha, \omega_{i}\right)$ defines an $\mathrm{SU}(2)$-structure on $N$. In particular, $e^{1}, \ldots, e^{5}$ is an orthonormal basis of 1-forms on $N$ : so, we are not using the quotient metric on the five-manifold, but a modified metric obtained by $\mathcal{D}$-homothety.

Conversely, suppose we have

$$
\left(N, \alpha, \omega_{1}, \omega_{2}, \omega_{3}, \phi, t\right),
$$

where $N$ is a five-manifold, $\left(\alpha, \omega_{i}\right)$ is an $\mathrm{SU}(2)$-structure on $N, t$ is a function on $N$ and $\phi$ is a closed two-form on $N$ such that

$$
\left[\frac{1}{2 \pi} \phi\right] \in H^{2}(N, \mathbb{Z}) .
$$

Let $M$ be a circle bundle over $N$ with Chern class $[\phi /(2 \pi)]$. Let $A$ be the standard generator of the Lie algebra $\mathfrak{u}(1)$, and let $X=A^{*}$ be the associated fundamental vector field. Choose a connection form $\eta$ such that $d \eta=\phi$ and define

$$
\begin{aligned}
& \omega=t^{-1} \omega_{3}+\eta \wedge \alpha, \\
& \Psi=\left(\omega_{1}+i \omega_{2}\right) \wedge\left(\eta+i t^{-2} \alpha\right) .
\end{aligned}
$$

It is clear that this defines an $\mathrm{SU}(3)$-structure preserved by $X$, which has norm $t \eta(X)=t$, and equations (15) give back the original $\mathrm{SU}(2)$-structure on $N$.

REMARK: The reduction we have chosen behaves well with respect to evolution theory. Indeed, let $N$ be a five-manifold with an SU(2)-structure; recall that $N$ is called hypo if the forms $\omega_{1}, \omega_{2} \wedge \alpha$, and $\omega_{3} \wedge \alpha$ are closed. It is easy to verify that the $\mathrm{SU}(3)$-structure induced on $N \times S^{1}$ by the above construction (corresponding to taking $\phi=0$ and $t=1$ ) is half-flat if and only if the structure on $N$ is hypo. Moreover, hypo geometry also has evolution equations similar to those mentioned in the introduction, and it turns out that a one-parameter solution of the hypo evolution equations lifts to a solution of the half-flat evolution equations. 
Recall from [4] that the intrinsic torsion of an SU(3)-structure takes values in a 42-dimensional space, and its components can be represented as follows:

\begin{tabular}{|c|c|}
\hline$W_{1}^{+}$ & $W_{1}^{-}$ \\
\hline$W_{2}^{+}$ & $W_{2}^{-}$ \\
\hline \multicolumn{2}{|c|}{$W_{3}$} \\
\hline \multicolumn{2}{|c|}{$W_{4}$} \\
\hline \multicolumn{2}{|c|}{$W_{5}$} \\
\hline
\end{tabular}

$\in$\begin{tabular}{|c|c|}
\hline $\mathbb{R}$ & $\mathbb{R}$ \\
\hline$\left[\Lambda_{0}^{1,1}\right]$ & {$\left[\Lambda_{0}^{1,1}\right]$} \\
\hline \multicolumn{2}{|c|}{$\llbracket \Lambda_{0}^{2,1} \rrbracket$} \\
\hline$\llbracket\left[\Lambda^{1,0} \rrbracket\right.$ \\
\hline$\llbracket \Lambda^{1,0} \rrbracket$ \\
\hline
\end{tabular}

meaning that the component $W_{1}^{+}$takes values in $\mathbb{R}$ and so on. Explicitly, we can write

$$
\begin{aligned}
& d \psi^{+}=\psi^{+} \wedge W_{5}+W_{2}^{+} \wedge \omega+W_{1}^{+} \omega^{2}, \\
& d \psi^{-}=\psi^{-} \wedge W_{5}+W_{2}^{-} \wedge \omega+W_{1}^{-} \omega^{2}, \\
& d \omega=-\frac{3}{2} W_{1}^{-} \psi^{+}+\frac{3}{2} W_{1}^{+} \psi^{-}+W_{3}+W_{4} \wedge \omega .
\end{aligned}
$$

We can do the same for SU(2)-structures on five-manifolds [6]; the intrinsic torsion now takes values in a 35-dimensional space, and we can arrange its components in the following table.

\begin{tabular}{|c|c|c|}
\hline \multicolumn{3}{|c|}{$\lambda$} \\
\hline$f_{1}$ & $f_{2}$ & $f_{3}$ \\
\hline$g_{1}^{2}$ & $g_{1}^{3}$ & $g_{2}^{3}$ \\
\hline \multicolumn{3}{|c|}{$\beta$} \\
\hline$\gamma_{1}$ & $\gamma_{2}$ & $\gamma_{3}$ \\
\hline \multicolumn{3}{|c|}{$\omega^{-}$} \\
\hline$\sigma_{1}^{-}$ & $\sigma_{2}^{-}$ & $\sigma_{3}^{-}$ \\
\hline
\end{tabular}

$\in$\begin{tabular}{|c|c|c|}
\hline \multicolumn{3}{|c|}{$\mathbb{R}$} \\
\hline $\mathbb{R}$ & $\mathbb{R}$ & $\mathbb{R}$ \\
\hline $\mathbb{R}$ & $\mathbb{R}$ & $\mathbb{R}$ \\
\cline { 2 - 3 } & \multicolumn{3}{|c|}{$\Lambda^{1}$} \\
\hline$\Lambda^{1}$ & $\Lambda^{1}$ & $\Lambda^{1}$ \\
\hline \multicolumn{3}{|c|}{$\Lambda_{-}^{2}$} \\
\hline$\Lambda_{-}^{2}$ & $\Lambda_{-}^{2}$ & $\Lambda_{-}^{2}$ \\
\hline
\end{tabular}

In the above table, $\Lambda^{1}$ is the four-dimensional representation of SU(2) such that the tangent space at a point is

$$
T=\Lambda^{1} \oplus \mathbb{R}
$$

whereas $\Lambda_{-}^{2}$ is the three-dimensional representation of SU(2) consisting of anti-self-dual two-forms on $\Lambda^{1}$. We shall write, say, $(\omega)_{\Lambda_{-}^{2}}$ for the $\Lambda_{-}^{2}$ component of a two-form $\omega$.

Setting $g_{i}^{j}=-g_{j}^{i}$, the components of the intrinsic torsion are given by

$$
\begin{aligned}
& d \alpha=\alpha \wedge \beta+\sum_{j=1}^{3} f^{j} \omega_{j}+\omega^{-} \\
& d \omega_{i}=\gamma_{i} \wedge \omega_{i}+\lambda \alpha \wedge \omega_{i}+\sum_{j \neq i} g_{i}^{j} \alpha \wedge \omega_{j}+\alpha \wedge \sigma_{i}^{-} .
\end{aligned}
$$


We can now prove the following.

Proposition 3.1 Define the intrinsic torsion of $M$ as above, and write

$$
\left.W_{i}=\eta \wedge \Xi_{i}+\Delta_{i}, \quad \text { where } \Xi_{i}=X\right\lrcorner W_{i} ;
$$

then the intrinsic torsion of the quotient is given as follows.

\begin{tabular}{|c|c|c|}
\hline \multicolumn{3}{|c|}{$-\left\langle\Delta_{5}, \alpha\right\rangle$} \\
\hline$\frac{3}{2} W_{1}^{-}$ & $-\frac{3}{2} W_{1}^{+}-\frac{1}{2}\left\langle\Xi_{3}, \omega_{2}\right\rangle$ & $-t^{-1} \Xi_{4}-\frac{1}{2}\left\langle\Xi_{3}, \omega_{3}\right\rangle$ \\
\hline$-t^{-2} \Xi_{5}$ & $-2 t^{-1} W_{1}^{+}-t^{-1}\left\langle\Xi_{2}^{+}, \alpha\right\rangle$ & $-2 t^{-1} W_{1}^{-}-t^{-1}\left\langle\Xi_{2}^{-}, \alpha\right\rangle$ \\
\hline \multicolumn{3}{|c|}{$\left.-\left(\Delta_{4}\right)_{\Lambda^{1}}-\alpha\right\lrcorner \Xi_{3}$} \\
\hline$\left.-\left(\Delta_{5}\right)_{\Lambda^{1}}-t^{-1} \Xi_{2}^{+}\right\lrcorner \omega_{2}$ & $\left.-\left(\Delta_{5}\right)_{\Lambda^{1}}+t^{-1} \Xi_{2}^{-}\right\lrcorner \omega_{1}$ & $\left.\left(\Delta_{4}+d \log t+\frac{1}{2} t \omega_{3}\right\lrcorner \Delta_{3}\right)_{\Lambda^{1}}$ \\
\hline \multicolumn{3}{|c|}{$-\left(\Xi_{3}\right)_{\Lambda_{-}^{2}}$} \\
\hline$-\Delta_{2}^{+}$ & $-\Delta_{2}^{-}$ & $\left.t(\alpha\lrcorner \Delta_{3}-\phi\right)_{\Lambda_{-}^{2}}$ \\
\hline
\end{tabular}

Proof. Taking the interior product of (18) with $X$, then substituting (15) in the left-hand side and (16) in the right-hand side, one easily computes

$$
\begin{gathered}
d \alpha=\frac{3}{2} W_{1}^{-} \omega_{1}-\frac{3}{2} W_{1}^{+} \omega_{2}-\Xi_{3}-t^{-1} \Xi_{4} \omega_{3}+\Delta_{4} \wedge \alpha, \\
d \omega_{1}=-\omega_{1} \wedge \Delta_{5}-t^{-2} \Xi_{5} \omega_{2} \wedge \alpha-t^{-1} \Xi_{2}^{+} \wedge \omega_{3}-\Delta_{2}^{+} \wedge \alpha-2 t^{-1} W_{1}^{+} \omega_{3} \wedge \alpha, \\
d \omega_{2}=-\omega_{2} \wedge \Delta_{5}+t^{-2} \Xi_{5} \omega_{1} \wedge \alpha-t^{-1} \Xi_{2}^{-} \wedge \omega_{3}-\Delta_{2}^{-} \wedge \alpha-2 t^{-1} W_{1}^{-} \omega_{3} \wedge \alpha .
\end{gathered}
$$

On the other hand, $\left.d \omega_{3}=d \log t \wedge \omega_{3}-t X\right\lrcorner d(\omega \wedge \eta)$ by (15). Using (18) and then (16), we obtain

$$
\begin{aligned}
d(\omega \wedge \eta)= & \frac{3}{2} t^{-2} W_{1}^{-} \omega_{2} \wedge \alpha \wedge \eta+\frac{3}{2} t^{-2} W_{1}^{+} \omega_{1} \wedge \alpha \wedge \eta+\Delta_{3} \wedge \eta \\
& +t^{-1} \Delta_{4} \wedge \omega_{3} \wedge \eta+t^{-1} \omega_{3} \wedge \phi+\eta \wedge \alpha \wedge \phi
\end{aligned}
$$

therefore

$$
d \omega_{3}=d \log t \wedge \omega_{3}+\frac{3}{2} t^{-1} W_{1}^{-} \omega_{2} \wedge \alpha+\frac{3}{2} t^{-1} W_{1}^{+} \omega_{1} \wedge \alpha+t \Delta_{3}+\Delta_{4} \wedge \omega_{3}-t \alpha \wedge \phi
$$

The decompositions (20) of the components $W_{3}$ and $W_{2}^{ \pm}$correspond to projections

$$
\llbracket \Lambda_{0}^{2,1} \rrbracket \rightarrow \omega_{1}^{\perp} \oplus\left(\alpha \wedge \omega_{1}\right)^{\perp}, \quad\left[\Lambda_{0}^{1,1}\right] \rightarrow T \oplus \Lambda_{-}^{2},
$$

respectively; the statement is now a straightforward consequence of (19).

REMARK: Of the decompositions (21), the first is not surjective; in other words, the components $\Xi_{3}$ and $\Delta_{3}$ are not independent.

It is clear that one can write down a converse to Proposition 3.1, because the quotient determines the intrinsic torsion of $M$; one can then characterize the $M$ with special intrinsic torsion in terms of 
the quotient. For example, in the symplectic half-flat case, one obtains the following generalization of Lemma 2.2.

PROPOSITION 3.2 M is symplectic half-flat if and only if the quotient satisfies

$$
\begin{aligned}
& d \alpha=0, \quad d \omega_{1}=0, \quad d \omega_{2} \wedge \alpha=t^{2} \omega_{1} \wedge \phi+2 d \log t \wedge \omega_{2} \wedge \alpha \\
& d \omega_{3}=d \log t \wedge \omega_{3}-t \alpha \wedge \phi
\end{aligned}
$$

Proof. This follows immediately from (16).

We now consider the case where $M$ is integrable. In order to state our theorem, we need to introduce two differential operators on five-manifolds with an SU(2)-structure. The first one is $\partial_{\alpha}$, which maps a function $f$ to $\langle\alpha, d f\rangle$. Secondly, consider the endomorphism $J_{3}$ of $T^{*} N$ characterized by

$$
J_{3} \alpha=0, \quad \omega_{1} \wedge \beta=\omega_{2} \wedge J_{3} \beta \quad \text { for } \beta \in \alpha^{\perp}
$$

we can then define an operator $d^{c}$ which maps a function $f$ to $d^{c} f=J_{3} d f$. We can now express the intrinsic torsion in terms of the norm $t$ of the Killing vector field.

THEOREM 3.3 If the SU(3)-structure on $M$ is integrable, $t$ is a solution of

$$
\partial_{\alpha}^{2} \log t-\left(\partial_{\alpha} \log t\right)^{2}-2 t^{-1}\left\|(d \log t)_{\Lambda^{1}}\right\|^{2}=0 .
$$

The intrinsic torsion is determined by $t$ as follows: $\alpha, \omega_{1}$ and $\omega_{2}$ are closed, and $\omega_{3}$ satisfies

$$
d \omega_{3}=(d \log t)_{\Lambda^{1}} \wedge \omega_{3}+\frac{1}{\partial_{\alpha} t} \alpha \wedge\left(2 d \log t \wedge d^{c} \log t-d d^{c} \log t\right)_{\Lambda_{-}^{2}}
$$

moreover the 'curvature form' is

$$
\phi=t^{-1} \partial_{\alpha} \log t \omega_{3}-\frac{1}{t^{2} \partial_{\alpha} \log t}\left(2 d \log t \wedge d^{c} \log t-d d^{c} \log t\right)_{\Lambda_{-}^{2}}-2 t^{-2} \alpha \wedge d^{c} \log t
$$

Conversely, let $N$ be a five-manifold with an $\mathrm{SU}(2)$-structure $\left(\alpha, \omega_{i}\right)$ and a function $t$, where $\alpha, \omega_{1}$ and $\omega_{2}$ are closed, and (23), (24) are satisfied. Then the two-form $\phi$ defined by (25) is closed; if the cohomology class $[\phi / 2 \pi]$ is an element of $H^{2}(N, \mathbb{Z})$, there is a circle bundle over $N$ on which an integrable SU(3)-structure is defined by (16), where $\eta$ is a connection form such that $d \eta=\phi$.

Locally, Theorem 3.3 is a characterization. Indeed, in the second part one can restrict $N$ to a contractible open subset $N^{\prime}$, so that

$$
0=[\phi / 2 \pi] \in H^{2}\left(N^{\prime}, \mathbb{Z}\right)
$$


Proof. It is clear from (15) that $\alpha, \omega_{1}$ and $\omega_{2}$ are closed. Hence, the only non-vanishing components of the intrinsic torsion are $\gamma_{3}$ and $\sigma_{3}^{-}$, determined by

$$
d \omega_{3}=\gamma_{3} \wedge \omega_{3}+\alpha \wedge \sigma_{3}^{-} .
$$

Using (16), we find

$$
t^{-1} \omega_{3} \wedge\left(\gamma_{3}-d \log t\right)+\alpha \wedge\left(t^{-1} \sigma_{3}^{-}+\phi\right)=0
$$

Hence $\gamma_{3}=(d \log t)_{\Lambda^{1}}$, and the component of $\phi$ in $\Lambda^{2}\left(\alpha^{\perp}\right)$ is determined by

$$
\left\langle\phi, \omega_{1}\right\rangle=0=\left\langle\phi, \omega_{2}\right\rangle, \quad\left\langle\phi, \omega_{3}\right\rangle=2 t^{-1} \partial_{\alpha} \log t, \quad(\phi)_{\Lambda_{-}^{2}}=-t^{-1} \sigma_{3}^{-} .
$$

It also follows from (16) that

$$
\begin{aligned}
& \omega_{1} \wedge \phi+2 t^{-3} d t \wedge \omega_{2} \wedge \alpha=0 \\
& \omega_{2} \wedge \phi-2 t^{-3} d t \wedge \omega_{1} \wedge \alpha=0
\end{aligned}
$$

Therefore

$$
\alpha\lrcorner \phi=-2 t^{-2} d^{c} \log t .
$$

For brevity, we set $s=\partial_{\alpha} \log t$. By construction $\phi$ is closed, so

$$
\begin{aligned}
0= & t^{-1}\left(-s d \log t \wedge \omega_{3}+d s \wedge \omega_{3}+s(d \log t-s \alpha) \wedge \omega_{3}+s \alpha \wedge \sigma_{3}^{-}\right. \\
& \left.+d \log t \wedge \sigma_{3}^{-} d \sigma_{3}^{-}\right)+2 t^{-2} \alpha \wedge\left(-2 d \log t \wedge d^{c} \log t+d d^{c} \log t\right)
\end{aligned}
$$

We can split (26) into two equations by taking the wedge and the interior product with $\alpha$. One of these is satisfied automatically: indeed, taking $d$ of $d \omega_{3}$ we find

$$
0=\alpha \wedge\left(d \log t \wedge \sigma_{3}^{-}-d \sigma_{3}^{-}+d s \wedge \omega_{3}\right)
$$

so the right-hand side of (26) vanishes on wedging with $\alpha$. Taking the interior product gives

$$
\left(\partial_{\alpha} s-s^{2}\right) \omega_{3}+2 s \sigma_{3}^{-}+2 t^{-1}\left(-2 d \log t \wedge d^{c} \log t+d d^{c} \log t\right)_{\Lambda^{2}\left(\alpha^{\perp}\right)} .
$$

It is now clear that $\sigma_{3}^{-}$can be expressed in terms of $t$, giving (24). Using the general formula

$$
\left\langle\beta \wedge J_{3} \beta, \omega_{3}\right\rangle=\|\beta\|^{2}-\langle\beta, \alpha\rangle^{2},
$$

we also deduce that $t$ satisfies (23).

Conversely, suppose (24) and (23) are satisfied, and define $\phi$ by (25). The above calculations show that $\phi$ is closed and (16) defines an integrable SU(3)-structure.

REMARK: The condition of Theorem 3.3 implies in particular that 28 out of the 35 components of the intrinsic torsion of $\left(N, \alpha, \omega_{i}\right)$ vanish. A similar construction was described in [1], starting with a seven-manifold with holonomy $\mathrm{G}_{2}$ and defining an $\mathrm{SU}(3)$-structure on the quotient. In that case, the vanishing components of the intrinsic torsion of the quotient are also 28, though out of 42 . 
In general, (23) and (24) are not independent, because the norm on one-forms depends on $\omega_{3}$. Motivated by this observation, we consider the special case

$$
(d \log t)_{\Lambda^{1}}=0
$$

In order to apply Theorem 3.3, we have to assume that the SU(2)-structure is integrable. Let $x$ be a coordinate in the direction of $\alpha$, so that $\alpha=d x$. Set $t=(1-x)^{-1}$; then (23) is satisfied. Suppose that one has a circle bundle over $N$ with Chern class $\left[1 / 2 \pi \omega_{3}\right]$; then the hypotheses of Theorem 3.3 hold. Define a connection form $\eta$ such that $d \eta=\omega_{3}$; then

$$
\omega=(1-x) \omega_{3}+\eta \wedge \alpha, \quad \Psi=\left(\omega_{1}+i \omega_{2}\right) \wedge\left(\eta+i(1-x)^{2} \alpha\right)
$$

defines an integrable SU(3)-structure. One can actually prove that if the original five-manifold has holonomy SU(2), then the Calabi-Yau six-manifold has holonomy SU(3).

\section{Acknowledgements}

This work was supported by the Project M.I.U.R. 'Geometric Properties of Real and Complex Manifolds' and by G.N.S.A.G.A. of I.N.d.A.M.

\section{References}

1. V. Apostolov and S. Salamon, Kähler reduction of metrics with holonomy $G_{2}$, Comm. Math. Phys. 246 (2004), 43-61.

2. L. Bedulli, Tre-varietà di Calabi-Yau generalizzate, Ph. D. Thesis, Università di Firenze, 2004.

3. S. Chiossi and A. Fino, Conformally parallel $G_{2}$ structures on a class of solvmanifolds, Math. Z. 252 (2006), 825-848.

4. S. Chiossi and S. Salamon, The intrinsic torsion of $\mathrm{SU}(3)$ and $G_{2}$ structures, Differential Geometry, Valencia 2001, World Scientific, 2002, 115-133.

5. S. Chiossi and A. Swann, $G_{2}$-structures with torsion from half-integrable nilmanifolds, J. Geom. Phys. 54 (2005), 262-285.

6. D. Conti and S. Salamon, Generalized Killing spinors in dimension 5, DG/0508375. Trans. Amer. Math. Soc., to appear.

7. P. de Bartolomeis and A. Tomassini, On solvable generalized Calabi-Yau manifolds, Ann. Inst. Fourier 56 (2006), 1281-1296.

8. P. de Bartolomeis and A. Tomassini, On the Maslov index of Lagrangian submanifolds of generalized Calabi-Yau manifolds, Int. J. Math. 17 (2006), 921-947.

9. D. Giovannini, Special structures and symplectic geometry, Ph. D. Thesis, Università degli studi di Torino, 2003.

10. N. Hitchin, The geometry of three-forms in six dimensions, J. Differential Geom. 55 (2000), 547-576.

11. N. Hitchin, Stable forms and special metrics, Global Differential Geometry: The Mathematical Legacy of Alfred Gray, Contemp. Math. 288, Amer. Math. Soc., Providence, 2001, 70-89.

12. A. Moroianu, P.-A. Nagy and U. Semmelmann, Unit Killing vector fields on nearly Kahler manifolds, Int. J. Math. 16 (2005), 281-301. 